\title{
Contributions of Sidewall Illumination and Current Spreading to the Light Emission of InGaN-GaN Light-Emitting Diode Arrays
}

\author{
Yi-Chen Yu, Chih-Hao Hsieh, Ghien-An Shih, Tzu-Yang Chiu, Dong-Ming Yeh, Chih-Feng Lu, \\ JianJang Huang, Member, IEEE, and C. C. Yang, Senior Member, IEEE
}

\begin{abstract}
We investigate the contribution of sidewall illumination in InGaN-GaN quantum-well-based light-emitting diode (LED) arrays with various cell radii. The intensity contribution from the array sidewall decreases with the increase of radius as the perimeter/area ratio is reduced. We then compare the effects of current spreading in the LED arrays of different sizes and conclude that the effect of current spreading needs to be given full consideration when the cell size in a microarray becomes larger. This letter provides a novel approach to calculate the intensity contributions of sidewall illumination and current spreading to GaN-based LED arrays.
\end{abstract}

Index Terms-Light-emitting diodes (LEDs), microarray, sidewall illumination.

\section{INTRODUCTION}

$\mathbf{T}$ HE demand for solid-state lighting for general illumination, backlights in flat-panel displays, and automotive lighting has pushed the development of high-power and highly efficient light-emitting diodes (LEDs). The low external extraction efficiency of LEDs is believed to be the bottleneck for high-power operations. More than $80 \%$ of light is totally reflected from the semiconductor-air interface in a conventional LED structure. To resolve the drawback, some groups have proposed various designs of surface texturing to roughen the surface for improving light extraction [1], [2]. Furthermore, the idea of microarray was demonstrated to achieve higher extraction efficiency than the conventional broad-area devices [3]-[6]. For example, Jin et al. [3] reported an interconnected microdisk with an efficiency improvement of around 60\%, when compared with conventional broad-area devices. Also, Huseh et al. [4] showed an around 40\% improvement by etching up holes in the p-type mesa. In addition, Choi et al. [5] have shown a $60 \%$ improvement with a microring array structure. They all attributed the efficiency improvement of such microarrays over the conventional broad-area structures to the scattering of light from the sidewall perimeter. In all of those devices, the efficiency decreases as the size of the cells in the microarrays becomes larger. Despite the demonstration

Manuscript received January 10, 2006; revised February 4, 2006. This work was supported by the National Science Council of Taiwan under Grant NSC 94-2215-E-002-028.

The authors are with the Graduate Institute of Electro-Optical Engineering and Department of Electrical Engineering, National Taiwan University, Taipei 106, Taiwan (e-mail: jjhuang@cc.ee.ntu.edu.tw).

Digital Object Identifier 10.1109/LPT.2006.873461 of improvement in the external quantum efficiency, the identification of the real contribution, either from the enhancement of light extraction or the increase of internal quantum efficiency, in such an LED array is still unavailable. The internal quantum efficiency improvement, especially from the current spreading in the p-type layer, is also a key factor for increasing the output power of an LED array as the cell size becomes larger. The considerations of both sidewall illumination and current spreading conditions in the mesa area are essential in designing an LED array structure.

In the letter, we fabricate both microdisk and microring LED arrays with various dimensions. We also develop a novel process for p-type metal interconnections in the microdisk and microring structures. The sidewall of each cell is covered with a thick interconnect metal to eliminate sidewall illumination. We then calculate the intensity contributions of the effects of sidewall illumination and current spreading of different microarrays of different cell sizes. The dimensions of LED arrays in this letter were designed to be much larger than previously published LED arrays in order to understand the upper limit of sidewall illumination.

\section{DEVICE FABRICATION}

The microring and microdisk samples were grown on $c$-plane sapphire substrates. The material structure is composed of $25 \mathrm{~nm}$ of GaN buffer layer, $1.8 \mu \mathrm{m}$ of Si-doped n-type GaN layer, a five-period InGaN-GaN multiple quantum wells, and a $0.2-\mu \mathrm{m}$ p-type $\mathrm{GaN}$ layer. The spectral peak wavelength of photoluminescence is around $470 \mathrm{~nm}$. The process of LED arrays started with inductively coupled plasma etching to define the mesa areas. We used $\mathrm{Ni}-\mathrm{Au}$ for $\mathrm{p}$-type and $\mathrm{Ti}-\mathrm{Al}$ for $\mathrm{n}$-type contacts. They were separately annealed to achieve ohmic contact. The p-type contact strip is $4 \mu \mathrm{m}$ in width and is $2 \mu \mathrm{m}$ away from the p-type mesa edge. On the subsequent steps, we first coated the sample with a $200-\mathrm{nm} \mathrm{SiN}_{x}$ insulating layer with plasma-enhanced chemical vapor deposition. The $\operatorname{SiN}_{x}$ layer is used to avoid the contact of the n-type GaN layer and the subsequent interconnect metal layer. Moreover, since the refractive index of $\operatorname{SiN}_{x}(n=2.0)$ is between that of the air and $\mathrm{GaN}$ semiconductor $(n=2.4$ ), the dielectric layer helps to increase light extraction. We then opened via holes on the $\mathrm{SiN}_{x}$ layer and evaporated a thick $\mathrm{Ti}(20 \mathrm{~nm}) / \mathrm{Au}(560 \mathrm{~nm})$ metal layer to connect microdisks/microrings p-type contact in the LED array. 
(a)

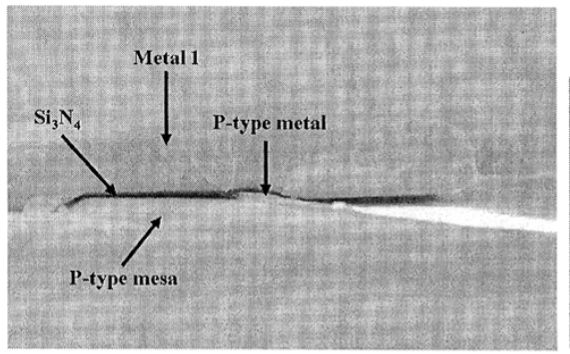

(c)

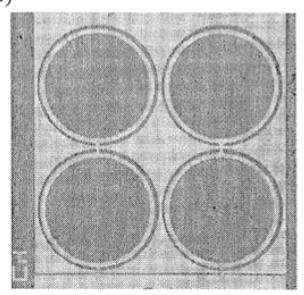

(b)

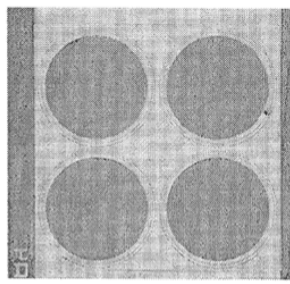

(d)

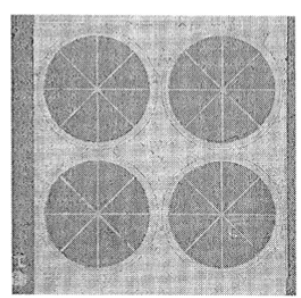

Fig. 1. (a) Cross-sectional view of microdisk with interconnect sidewall metal; (b) four-cell microdisk with sidewall metal (E-100-04); (c) four-cell microdisk (B-100-04); (d) four-cell microdisk with sidewall metal and star-shape p-type contacts/ITO layer (C-100-04).

TABLE I

NOMENCLATURE OF MICROARRAY SAMPLES

\begin{tabular}{|l|l|l|l|l|l|}
\hline Structure & $\begin{array}{l}\text { Micro-ring } \\
(1)\end{array}$ & $\begin{array}{l}\text { Micro-disk } \\
(1)\end{array}$ & $\begin{array}{l}\text { Micro-disk } \\
(2)\end{array}$ & $\begin{array}{l}\text { Micro-ring } \\
(2)\end{array}$ & $\begin{array}{l}\text { Micro-disk } \\
(3)\end{array}$ \\
\hline Sidewall metal & N/A & N/A & V & V & V \\
\hline $\begin{array}{l}\text { Star shape p-type } \\
\text { contact/ITO } \\
\text { layer }\end{array}$ & N/A & N/A & V & N/A & N/A \\
\hline Sample & A-xxx-yy & B-xxx-yy & C-xxx-yy & D-xxx-yy & E-xxx-yy \\
\hline $\begin{array}{l}\text { xxx denote radius (in } \mu \text { m) of each cell and yy denote cell numbers in a array. For } \\
\text { instance, B-100-04 represents 4-cell micro-disk with radius 100 } \mu \text { m. }\end{array}$ \\
\hline
\end{tabular}

To study the microarray illumination, we designed five types of microdisk and microring structures. Microarrays, with sidewalls either covered with thick interconnect metal or not, were fabricated for comparing the contributions from sidewall illumination. The scanning electron microscopy image in Fig. 1(a) shows a microdisk with its sidewall covered with metal. The slope of the p-type mesa is approximately $75^{\circ}$ with respect to the wafer surface that ensures the complete elimination of sidewall optical output since most light reflected from the covered interconnect metal will not reach the p-type mesa surface. We also fabricated microarrays with and without star-shaped p-type contacts and indium tin oxide (ITO) layer to understand the effects of current spreading. For those samples with star-shaped contacts, we sputtered and alloyed an additional 200-nm ITO layer on top of the p-type mesa to enhance current spreading prior to the deposition of $\operatorname{SiN}_{x}$. The 4- $\mu \mathrm{m}$ width star-shaped p-type metal strips were first coated to improve carrier flow across the ITO layer without blocking too much light. The nomenclature of those microarray structures is described in Table I. The pictures of four-cell microdisks are shown in Fig. 1(b), (c), and (d).

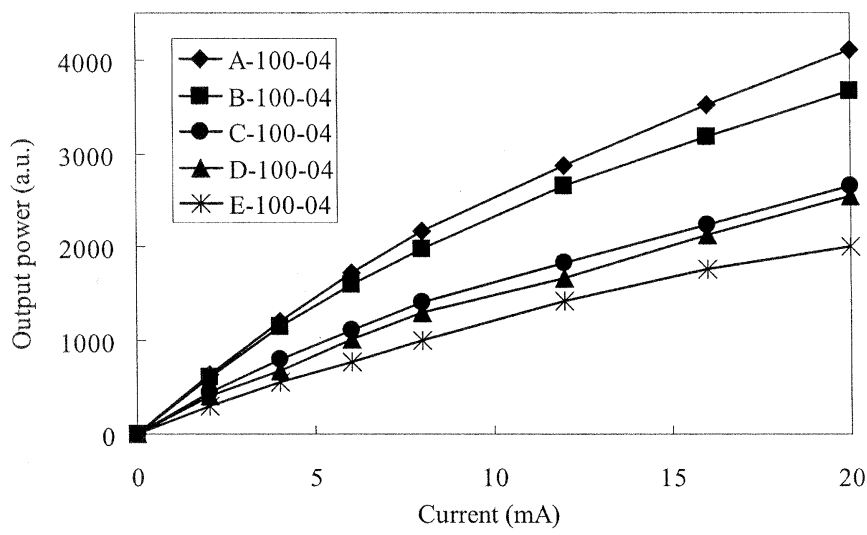

Fig. 2. Light output-current $(L-I)$ curves of several types of microarrays under investigation.

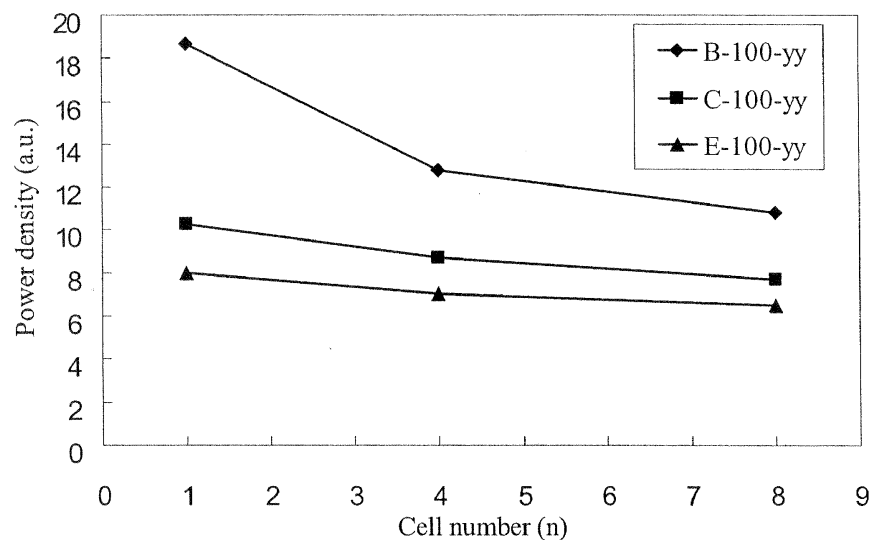

Fig. 3. Output power densities of one-, four-, eight-cell microdisks.

\section{CHARACTERIZATIONS OF MICROARRAYS}

To compare the output power of those five types of microarrays, output powers as functions of injection current of samples A, B, C, D, and E, all with four cells in an array and cell radius $100 \mu \mathrm{m}$, were measured. As shown in Fig. 2, the sidewall illumination plays an important role in contributing total optical output power. The microring structure has the highest output power while the microdisk with sidewall covered with thick metal has the lowest one. In addition, the star-shaped p-type metal contact along with the ITO layer help spread out injected currents. Thus, the output power is higher than sample C-100-04 than that of sample E-100-04.

The dependence of the output power density, defined as the output power per unit p-type mesa area, on cell numbers of microdisk arrays is plotted in Fig. 3. The injected current density is fixed at $0.08 \mu \mathrm{A} / \mu \mathrm{m}^{2}$ for all of the microarrays under consideration and the radius of each cell is $100 \mu \mathrm{m}$. Microdisks covered with sidewall metal show only little decrease of power densities as the cell number increases. The decrease is attributed to a slightly higher series resistance of the p-type metal contact as the cell number increases. We believe the decrease is even more severe as part of the current density is used for sidewall illumination. A decline is found for microdisks without sidewall metal (samples B-100-yy). 
(a)

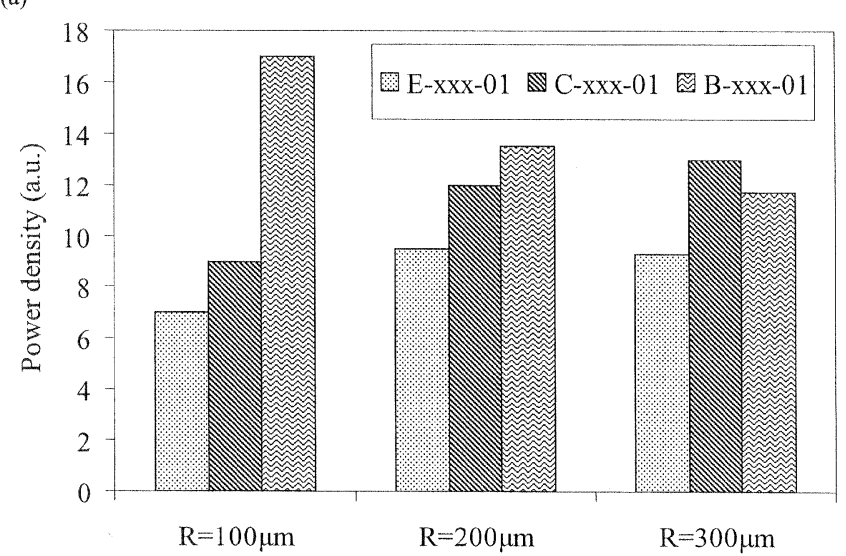

(b)

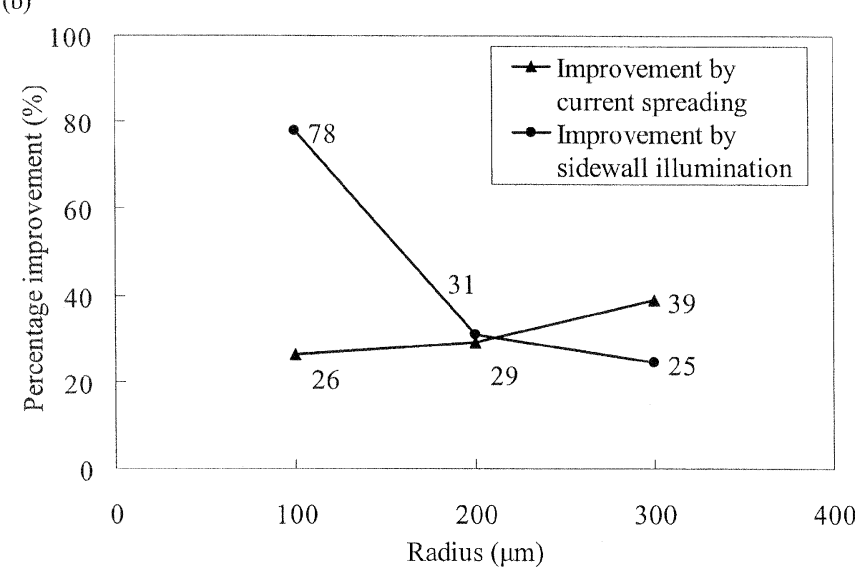

Fig. 4. (a) Power densities of one-cell LEDs with radius 100, 200, and $300 \mu \mathrm{m}$. (b) Contributions of sidewall illumination and current spreading [from (a)] at different cell size.

We next compare the output power with different cell dimensions. As shown in Fig. 4(a), sidewall illumination enhances the light extraction out of a one-cell LED under the same bias current density $\left(0.07 \mu \mathrm{A} / \mu \mathrm{m}^{2}\right)$. The current spreading $(C-x x x-01)$ is not as significant as sidewall illumination when the cell radius is within $200 \mu \mathrm{m}$. However, the current spreading becomes critical when the cell radius is larger. For radius $300 \mu \mathrm{m}$, the contribution from sidewall illumination is smaller than the effect of current spreading since the aspect ratio (perimeter/area) is smaller. Proper current spreading in the large microdisk area becomes important.
Contributions of sidewall illumination and microdisk current spreading of those samples in Fig. 4(a) are plotted in Fig. 4(b). For devices with radius $100 \mu \mathrm{m}$, sidewall illumination enhances optical output by $78 \%$ when compared with devices that the sidewall is completely blocked by thick metal (E-100-01). It then decreases to $25 \%$ as the radius is $300 \mu \mathrm{m}$. In contrast, the improvement of efficiency by the effect of current spreading becomes more significant than that by the sidewall illumination. Sample C-300-01 has 39\% more output power than sample E-300-01.

\section{CONCLUSION}

The illumination of LED microarrays is affected by several factors such as sidewall illumination, microdisk area, and current spreading. We compare the optical output power of several types of microarray structures. The ratio of sidewall illumination to total output power can be calculated by comparing microarrays with and without sidewall metal. The effect of current spreading becomes more significant than sidewall illumination as the cell size becomes larger. The improvement of efficiency by the effect of current spreading is $39 \%$ while the sidewall contribution is $25 \%$ for cell radius $300 \mu \mathrm{m}$. This letter provides a novel approach to calculate the intensity contributions of sidewall illumination and current spreading to GaN-based LED arrays.

\section{REFERENCES}

[1] C. M. Tsai, J. K. Sheu, W. C. Lai, Y. P. Hsu, P. T. Wang, C. T. Kuo, C. W. Kuo, S. J. Chang, and Y. K. Su, "Enhanced output power in GaN-based LEDs with naturally texture surface grown by MOCVD," IEEE Electron Device Lett., vol. 26, no. 7, pp. 464-466, Jul. 2005.

[2] K.-M. Chang, C.-H. Li, B.-S. Sheih, J.-Y. Yang, S.-W. Wang, and T.-H. Yeh, "A new simple and reliable method to form a textured Si surface for the fabrication of a tunnel oxide film," IEEE Electron Device Lett., vol. 19, no. 5, pp. 145-147, May 1998.

[3] S. X. Jin, J. Li, J. Y. Lin, and H. X. Jiang, "InGaN/GaN quantum well interconnected microdisk," Appl. Phys. Lett., vol. 77, no. 20, pp. 3236-3238, Nov. 2000.

[4] T. H. Hsueh, J. K. Sheu, H. W. Huang, J. Y. Chu, C. C. Kao, H. C. Kuo, and S. C. Wang, "Enhancement in light output of InGaN-based microhole array light-emitting diodes," IEEE Photon. Technol. Lett., vol. 17, no. 6, pp. 1163-1165, Jun. 2005.

[5] H. W. Choi, C. W. Jeon, and M. D. Dawson, "InGaN microring lightemitting diodes," IEEE Photon. Technol. Lett., vol. 16, no. 1, pp. 33-35, Jan. 2004.

[6] H. W. Choi, M. D. Dawson, P. R. Edwards, and R. W. Martin, "High extraction efficiency InGaN micro-ring light-emitting diodes," Appl. Phys. Lett., vol. 83, no. 22, pp. 4483-4485, Dec. 2000. 